\title{
LONG TIME BEHAVIOR OF PARTICLE SYSTEMS IN THE MEAN FIELD LIMIT
}

\author{
EMANUELE CAGLIOTI* AND FRÉDÉRIC ROUSSET ${ }^{\dagger}$
}

\begin{abstract}
We present a review of some recent results concerning the long time behavior of particle systems in the mean field limit. In particular we will consider the Vlasov limit for a system of particles interacting via a two body potential, the case of the vortex model, and the case of the piston. In all these cases the particle system is described, in the mean field limit, by a suitable nonlinear Liouville equation. The main problem we are interested in is the comparison between the limit dynamics and the behavior of the particle system when $N$ is large.
\end{abstract}

Key words. Vlasov type equations, particles systems, stability.

AMS subject classifications. $82 \mathrm{C} 22,82 \mathrm{C} 05$

\section{Introduction}

Many systems of particles are described, in the mean field limit, by a suitable nonlinear Liouville Equation: i.e. by a Liouville equation for a suitable phase space density $f$, in which the hamiltonian is a suitable functional of $f$ itself.

This is the case, for instance, of the mean field limit for a system of interacting particles which is described by the Vlasov equation; of the vortex model in the mean field limit which is described by the 2D Euler Equations; and of the piston which, in a suitable hydrodynamical limit, is described by a Vlasov-like equation with a singular interaction.

In general the validity of the approximation is proved for finite times or at most for times of the order of $\log N$, where $N$, is the number of particles.

A very interesting problem concerns the long time behavior of these systems. In particular one would like to write down the $1 / N$ corrections to the asymptotic Vlasov-like dynamics.

Here we briefly summarize some physical results on this problem and then we consider the following simpler but strictly related problem from a rigorous point of view.

If the particles are initially extracted from a stationary stable distribution of the nonlinear Liouville equation, for how long a time does the particles' distribution remain close to the initial one?

In Section 2 we will consider in some details the Vlasov case, reviewing some rigorous results on the subject and summarizing briefly some recent attempts to write down corrections to the Vlasov equation. In sections 3 and 4, respectively, we will briefly consider the case of the vortex model and of the piston.

\section{The Vlasov case}

A system of particles $x_{1}, v_{1}, \ldots, x_{N}, v_{N}$, interacting via a two-body potential $V$, in the mean-field limit, is described by the Vlasov equation

$$
\partial_{t} f+v \cdot \nabla_{x} f+F\left[\rho_{f}\right] \cdot \nabla_{v} f=0
$$

\footnotetext{
*Dipartimento di matematica, Universita di Roma I, La Sapienza, P.le Aldo Moro, 2. 00185 Roma, Italia (caglioti@mat.uniroma1.it).

${ }^{\dagger}$ CNRS, Laboratoire Dieudonne, Universite de Nice, Parc Valrose 06108 Nice Cedex 02, France (frousset@math.unice.fr).
} 
where $f(t, x, v) \in \mathbb{R}, x \in \mathbb{T}^{m}, v \in \mathbb{R}^{m}$, and the density $\rho_{f}$ and the force $F\left[\rho_{f}\right]$ are given by

$$
\rho_{f}(t, x)=\int_{v} f(t, x, v) d v, F\left[\rho_{f}\right](t, x)=-\nabla V \star \rho_{f}=-\int_{y} \nabla V(x-y) \rho_{f}(t, y) d y .
$$

More precisely, if $x_{1}, v_{1}, \ldots, x_{N}, v_{N}$, satisfy

$$
\begin{aligned}
\dot{x_{i}} & =v_{i}, \\
\dot{v_{i}} & =\frac{1}{N} \sum_{j=1}^{N} F\left(x_{i}-x_{j}\right)
\end{aligned}
$$

then (here we consider the case in which the force $F$ is Lipschitz)

$$
\mu_{N}(t)=\frac{1}{N} \sum_{k=1}^{N} \delta_{\left(x_{k}(t), v_{k}(t)\right)}
$$

is a weak solution of 2.1 .

Moreover the following classical result holds (see, for instance, [36]) :

TheOREM 2.1. Assume that $\nabla V$ is Lipschitz, then there exists $C>0, \kappa>0$ such that for any bounded measures $\mu_{1}^{0}, \mu_{2}^{0}$, the two measure solutions of (2.1) $\mu_{1}(t), \mu_{2}(t)$ with initial data $\mu_{1}^{0}$ and $\mu_{2}^{0}$ enjoy the estimate

$$
\left\|\mu_{1}(t)-\mu_{2}(t)\right\|_{L i p} \leq C e^{\kappa t}\left\|\mu_{1}^{0}-\mu_{2}^{0}\right\|_{L i p}, \forall t \geq 0,
$$

Where the bounded Lipshitz norm of measures is defined by

$$
\|\mu\|_{\text {Lip }}=\sup _{\varphi \in L i p,\|\varphi\|_{L i p} \leq 1}<\mu, \varphi>.
$$

The distance on the probability measures induced by this norm is equivalent to the Wasserstein distance and in particular metricizes the weak convergence.

Consequently, this Theorem yields that for finite times if $\mu_{N}$ converge to $\mu_{0}$ in the bounded Lipshitz norm (equivalent to the weak convergence) then also $\mu_{N}(t)$ converges to $\mu(t)$ in the same norm.

This also immediately implies that the particle system will remain close to the solution of the Vlasov equation for time of the order of $\log N$.

Before the description of the stability results, let us briefly summarize some recent physical results on the problem.

The problem of describing the finite $N$ corrections to the Vlasov Equation has been widely considered in the physical literature.

In particular in [37], [3], [4], the Hamiltonian Mean-Field Model (HFM) has been studied. In this case particles on a circle interact via the repulsive mean-field force $F=$ $\sin (x-y)$. In [37] the scenario described above is proposed and analyzed. Furthermore, from numerical simulations, there is evidence of the fact that the system converges to the thermodynamic equilibrium in times $T_{N}$ of order $N^{\alpha}$, with the non-trivial exponent $\alpha \simeq 1.7$.

In [4], by an analysis of the Vlasov equation linearized around a stable stationary solution, the fact that $T_{N}$ should be larger than $N$ is justified, while in [3] a Fokker Planck equation for the momentum of a particle in an equilibrium bath is derived. 
Let us now describe our stability results.

We focus now on the one-dimensional case $(m=1)$ to simplify the presentation, even if the results are actually valid in the general case. We consider a stationary distribution $f_{0}(v) \geq 0$, compactly supported, which is a stationary solution of the Vlasov equation. At the initial time we can choose randomly the particles $\left(x_{i}^{0}, v_{i}^{0}\right)_{i \in \mathbb{N}}$ with respect to the probability measure $\otimes_{\mathbb{N}} f_{0}(v) d x d v$ (we also assume that $\left.\int_{x, v} f_{0}(v) d x d v=1\right)$. For every $\alpha \in(0,1 / 2)$, there exists $C>0$ such that for almost every choice of the particles, we have the estimate

$$
\left\|\mu_{N}\left[X^{0}\right]-f_{0} d x d v\right\|_{L i p} \leq \frac{C}{N^{\frac{1}{2}-\alpha}},
$$

where

$$
\mu_{N}\left[X^{0}\right]=\frac{1}{N} \sum_{i=1}^{N} \delta_{\left(x_{i}^{0}, v_{i}^{0}\right)}
$$

The use of Theorem 2.1 gives that the empirical measure $\mu_{N}(t)$ of the particles evolving according to $(2.1),(2.3)$, with initial value $\left(x_{i}^{0}, v_{i}^{0}\right)$ will stay close to $f_{0}(v) d x d v$ in the bounded Lipschitz norm of measures on a time scale of order $\ln N$. When the distribution $f_{0}$ is a stable distribution of (2.1), we expect this time scale to be much longer. Indeed, we have proven in [6], [7] :

TheOREM 2.2. Assuming that $\partial_{x} V \in \mathcal{C}^{1,1}$ and that $\hat{V}_{l} \geq 0$ where $\hat{V}_{l}$ are the Fourier coefficients of $V$, we have for some $\beta>0$,

$$
\left\|\mu_{N}(t)-f_{0} d x d v\right\|_{L i p} \leq C N^{\beta}, \quad \forall t \in\left[0, T_{N}\right]
$$

where $T_{N}$ is given by

- if $f_{0}=g\left(v^{2} / 2\right)$ with $g \in \mathcal{C}^{2}$ and $g$ nonincreasing, $T_{N} \geq C N^{\frac{1}{8}-\eta}$,

- if $f_{0}=g(|v|)$, and $g \in L^{\infty}$, is nonincreasing, $T_{N} \geq C N^{\frac{1}{14}}-\eta$ and if moreover Leb $\left\{f_{0}=\left\|f_{0}\right\|_{\infty}\right\}>0$ then $T_{N} \geq N^{\frac{1}{10}-\eta}$

where $\eta>0$ can be chosen arbitrarily small.

The complete proof of this Theorem can be found in [6], [7]. The expression of $\beta$ is given explicitly. The monotonicity property of $f_{0}$ is the classical Penrose criterion of stability. Note that this Theorem is purely deterministic, the particles are just extracted randomly at the initial time, and then we use a stability result for the equation. With this kind of argument, we cannot go beyond the time scale $N^{\frac{1}{2}}$. We refer to [6] for an explicit counterexample. In particular it is easy to see that it is possible to choose initial conditions for the particle system whose distance from an homogeneous solution is of order $N^{-1 / 2}$ which at a time of the order of $N^{1 / 2}$ are at a distance of order 1 from this solution; see [6] for an explicit counterexample. Similar counterexamples can be constructed also in the interacting case. In particular in [14] Nonlinear Landau Damping has been proven for a suitable class of initial data. As a consequence(see section 4), one can show that initial data for the Vlasov-Poisson equation close in the bounded Lipschitz norm to a stationary stable solution, evolve, after a suitable time, far from it.

If $f_{0}$ is $\mathcal{C}^{2}$, the proof relies on the Energy-Casimir method that is the conservation of the quantity

$$
H_{C}(f)=\frac{1}{2} \int_{x, v} f v^{2} d x d v+\frac{1}{2} \int_{x} \rho_{f}(x) V\left[\rho_{f}\right](x) d x+\int_{x, v} q(f) d x d v
$$


for every function $q$ where

$$
\rho_{f}(x)=\int_{v} f d v, \quad V\left[\rho_{f}\right](x)=\int_{x} V(x-y) \rho_{f}(y) d y .
$$

The idea of the method is to choose $q$ in a suitable way so that $f_{0}$ becomes a nondegenerate extremum of the functional. This idea was introduced by Arnold in hydrodynamics [1] and also very much used in plasma physics; see [21], [34] for example. In plasma physics, this method is also known as the relative entropy method; see [2] for example. Since here we are studying a measure solution $\mu$, this quantity, which is very useful to prove stability results when we are dealing with functions, is not well-defined. The main idea is to use a duality argument to get that every measure solution of the Vlasov equation enjoys for every $T>0$, the estimate

$$
\|\mu(T)\|_{\text {Lip }} \leq\|\mu(0)\|_{L i p}\left\|R^{T}[\mu]\right\|
$$

where

$$
\left\|R^{T}[\mu]\right\|=\sup _{\|g\|_{L^{1}(0, T, L i p)} \leq 1}\left\|R^{T}[\mu](g)\right\|_{L i p}
$$

where for every $g \in L^{1}(0, T, \operatorname{Lip}), R^{T}[\mu](g)=\varphi(0, x, v)$ with $\varphi(t, x, v)$ the solution of the equation

$$
L^{*} \varphi+F[\mu] \partial_{v} \varphi=g, \quad F[\mu]=-<\mu(t), \partial_{x} V(x-\cdot)>,
$$

with the final condition $\varphi(T, x, v)=0$, and where $L^{*}$ is the adjoint of $L$, the linearized Vlasov operator about $f_{0}$ :

$$
L f=\partial_{t} f+v \partial_{x} f+F \partial_{v} f_{0}, \quad F=-\int_{x, v} \partial_{x} V(x-y) f(y, v) d v d y
$$

There are two steps in the estimate of $\left\|R^{T}[\mu]\right\|$. The first one is to get a good estimate of

$$
\int_{x, v}\left|g_{0}^{\prime}\right| \varphi^{2} d x d v
$$

This is done by constructing a functional which is conserved by $L^{*}$. This functional is deduced from the conservation of the Hamiltonian-Casimir by the direct equation. The second step is to use this last weighted $L^{2}$ norm to deduce a Lipschitz estimate on $\varphi$ thanks to the characteristics method.

If $f_{0}$ is merely $L^{\infty}$, the previous method fails and the proof of the estimate relies on the Marchioro-Pulvirenti method [28] . In this paper the stability of perturbations $f(t)$ of $f_{0}$ is obtained by using two ingredients. The first one is that $f$ is conserved along the characteristic flow of the Vlasov equation (2.1)

$$
\dot{x}=v, \quad \dot{v}=F
$$

which is measure preserving. The second one is that the minimum of the kinetic energy among the functions $f$ which have level sets of same measure as $f_{0}$ is reached at $f_{0}$. The fact that the total energy is a Lyapunov functional gives a control of the kinetic energy by the positivity of the potential energy, and this allows us to get the 
stability result. This powerful argument cannot be applied in the case where $f=\mu$ is a measure (a sum of dirac masses) since we cannot define the values of $\mu$. To overcome this difficulty, we consider the force created by the particles

$$
F(t, x)=-\frac{1}{N} \sum_{i=1}^{N} \partial_{x} V\left(x-x_{i}(t)\right)
$$

as an external forcing. Then, we consider the solution $f$ of the transport equation

$$
\partial_{t} f+v \partial_{x} f+F(t, x) \partial_{v} f=0
$$

with initial value $f_{0}$. This evolution preserves the measure of the level sets but not the energy. Nevertheless, as long as the force $F$ remains small, the empirical measure of the particles remains close to $f$ in the bounded Lipschitz norm of measure. Moreover, for the particles the total energy is conserved. This allows us to get a stability result by a perturbation of the Marchioro Pulvirenti argument.

\section{The vortex model}

We consider a regularized version of the vortex model: we consider the equation

$$
\partial_{t} \omega+u \cdot \nabla \omega=0
$$

$u$ is given by

$$
u=K \star \omega, \quad K=\nabla^{\perp} V(|x|),
$$

where $V(|x|)=\ln (|x|) \chi(|x|)$ with $\chi$ a smooth function which vanishes in a vicinity of the origin to remove the singularity. This equation is therefore a regularized version of the Euler equation in vorticity form. The particle system associated to this model is

$$
x_{i}^{\prime}(t)=\frac{1}{N} \sum_{j \neq i} K\left(x_{i}(t)-x_{j}(t)\right), i=1, \cdots, N .
$$

For measure solutions of (3.1), the same result as Theorem 2.1 is available when $V$ is Lipschitz (see [29] page 186 for example).

The convergence of the true vortex model (without smoothing) to the 2D Euler Equations is not an easy task due to the singularity of the interaction between the vortices. It was proven in [17] that it is possible to prove the convergence for a special choice of the particles at the initial time which is natural in numerical analysis (particles on a regular grid).

In this setting, we are still interested in the time evolution of $N$ particles extracted from a stationary stable distribution of $(3.1) \omega_{0} \geq 0$, which is compactly supported. In particular, it would be interesting to know if asymptotically the particles system reaches the microcanonical distribution $[22,27,8,9,24,32]$, and how this distribution can be reached. In a recent paper, [10], an equation has been proposed to describe the $1 / N$ correction to the $2 \mathrm{D}$ Euler Equations for the vortex model. This term would give a non-trivial evolution on the set of stationary solution of the Euler equations and is proportional to $1 / N$.

For what concerns an estimate of the time in which the system remains close to the initial distribution we are able to prove that: 
Theorem 3.1. Assuming that $K \in \mathcal{C}^{1,1}$, we have for some $\beta>0$ the estimate

$$
\left\|\omega_{N}(t, d x)-\omega_{0}\right\|_{L i p} \leq C N^{\beta}, \quad \forall t \in\left[0, T_{N}\right]
$$

with

$$
\omega_{N}(t, d x)=\frac{1}{N} \sum_{i=1}^{N} \delta_{x_{i}(t)}
$$

where $T_{N}$ is given by

- if $\omega_{0}(x)=\Omega\left(|x|^{2}\right)$ with $\Omega \in \mathcal{C}^{2}$ and $\Omega$ nonincreasing, $T_{N} \geq N^{\frac{1}{8}-\eta}$,

- if $\omega_{0}(x)=\Omega(|x|)$ with $\Omega \in L^{\infty}$ and $\Omega$ nonincreasing, $T_{N} \geq N^{\frac{1}{10}}-\eta$

Again, we can give an explicit expression of $\beta$. The proof follows the same lines as for the Vlasov case. In particular, we use the Energy-Casimir method in the first case and the Marchioro-Pulvirenti argument in the second case. The part of the total energy in the Vlasov case is played by the moment of inertia

$$
M(\omega(t))=\int \omega(t, x)|x|^{2} d x
$$

\section{The piston}

The time evolution of a system consisting of a piston of mass $M$ moving parallel to the $x$-axis in a cube of size $L$ containing $N=L^{3}$ non-interacting point particles of unit mass has been studied extensively [11, 12, 13, 15, 16, 18, 20, 23, 25, 26, 33]. By rescaling space and time by $L$ the problem reduces to that of a one-dimensional system with $N / 2$ particles in both the intervals $[0, X],[X, 1]$ where $X(t)$ is the position of the piston. The left (right) particles move freely between collisions with the wall at $x=0(x=1)$ and the piston at $x=X(t)$. When a particle collides with one of the two walls its velocity is reversed. The collision with the piston, which is also elastic, is described by the following collisions rules:

$$
\begin{aligned}
v^{\prime} & =-(1-\epsilon) v+(2-\epsilon) V \\
V^{\prime} & =\epsilon v+(1-\epsilon) V
\end{aligned}
$$

where $\epsilon=\frac{2}{M+1}, V=\dot{X}(t)$ and $V^{\prime}$ are the velocity of the piston before and after the collision, respectively, and $v, v^{\prime}$ are the velocities of the particle before and after the collision, repectively. The collisions preserve the kinetic energy, $\frac{1}{2} \sum_{i=1}^{N} v_{i}^{2}+\frac{1}{2} M V^{2}$, while the momentum is not preserved by the collisions with the walls at $x=0$ and $x=1$.

In $[25,11,12]$ this model was studied in the case where $M \sim N^{2 / 3}$, and the velocity of the particles is of order 1 . In the limit $N \rightarrow \infty$, see $[11,12]$, this system is described by a nonlinear Liouville equation with a singular interaction term.

We denote by $f(x, v)$ the phase space density and by $X$ and $V$ the position and the velocity of the piston, respectively. Out of the collisions set $(x=0, x=1, x=X)$ $f$ evolve freely:

$$
\partial_{t} f+v \partial_{x} f=0
$$

We can rewrite $(4.2)$ as

$$
f(x, v, t)=f(x-v(t-\tau), v, \tau)
$$


for any $x, v, \tau<t$, such that there is no collisions of the trajectory with the walls or with the piston. On the walls, $f$ satisfies the following boundary conditions:

$$
\begin{aligned}
& f(0, v)=f(0,-v) \text { for } v>0 ; \\
& f(1, v)=f(1,-v) \text { for } v<0 ;
\end{aligned}
$$

while the boundary conditions on the piston are:

$$
\begin{aligned}
& f\left(X^{-}, v\right)=f\left(X^{-}, 2 V-v\right) \text { for } v<V \\
& f\left(X^{+}, v\right)=f\left(X^{+}, 2 V-v\right) \text { for } v>V .
\end{aligned}
$$

Finally the velocity of the piston is determined by the condition $P_{L}(V)=P_{R}(V)$, where $P_{L}$ and $P_{R}$ denote the pressure exerted on the piston by the particles of the left and right compartment, respectively:

$$
\begin{aligned}
& P_{L}(V)=2 \int_{v>V} d v f\left(X^{-}, v\right)(v-V)^{2}, \\
& P_{R}(V)=2 \int_{v<V} d v f\left(X^{+}, v\right)(v-V)^{2} .
\end{aligned}
$$

The proof in $[25,11,12]$ is valid for a time interval in which any (almost any) particle does not collide with the piston more than twice.

For this Vlasov-like equation there exists a class of stationary stable solutions similar to the one described here.

More precisely, for any $\bar{X} \in(0,1)$, and nonnegative nonincreasing bounded functions $g_{L}(|v|), g_{R}(|v|)$ with finite kinetic energy and such that

$$
\int d v g_{L}(|v|)=\int d v g_{R}(|v|) \quad \text { (pressure balance), }
$$

$X=\bar{X}$ and

$$
f= \begin{cases}g_{L}(|v|) & \text { if } x \in(0, \bar{X}) \\ g_{R}(|v|) & \text { if } x \in(\bar{X}, 1)\end{cases}
$$

is a stable stationary solution of (4.2), see [5].

According to the numerical simulations, if particles at time $t=0$ are extracted from a stationary stable solution, see $[13,5]$, (different from the global Maxwellian equilibrium), then they remain close to it for a time of order $N^{\alpha}$, where $\alpha \in[2 / 3,5 / 6]$. Moreover, on the same time scale the $\mathrm{N}$-particles density converge, to the Maxwellian distribution.

In [19] the approach to the equilibrium has been studied for large values of $N$, and $M>>1$. In particular the system is found to approach the equilibrium in a time of the order of the mass of the piston $M$ and an equation for the motion of the piston on this time scale is derived.

A similar behavior has been observed in [30] for a gas of hard disks interacting with the piston. In particular in this paper time correlation function for the position of the piston has been computed by means of molecular dynamics simulations.

The case in which $N$ is finite and $M \rightarrow \infty$ has been studied in [35]. In this paper it has been proved that on a time scale of the order of $\sqrt{M}$ the piston behaves as a particle in a given potential field whose form is determined by the initial data. In [31] 
the same limit has been considered for a general gas. Under an ergodic hypothesis on the particles of the gas a similar picture has been rigorously derived.

The stability analysis performed in the present paper in the case of the Vlasov equation and of the regularized 2D Euler Equations use some regularity properties of these dynamics. The case of the piston is much more singular than the one considered here; nevertheless we have some hope that using a technique similar to the one in [7] it is possible to prove a similar result.

\section{REFERENCES}

[1] V. Arnold, Sur la géométrie différentielle des groupes de Lie de dimension infinie et ses applications à l'hydrodynamique des fluides parfaits, Ann. Inst. Fourier (Grenoble), 16(fasc. 1), 319-361, 1966.

[2] N. Benabdallah and J. Dolbeault, Relative entropies for kinetic equations in bounded domains irreversibility, stationary solutions, uniqueness, Arch. Ration. Mech. Anal., 168, 253-298, 2003.

[3] F. Bouchet, The stochastic process of equilibrium fluctuations, of a system with long range interactions, Phys. Rev. E, 70, 036113, 2004.

[4] F. Bouchet and T. Dauxois, Out-of-equilibrium relaxation and anomalous correlations of the HMF model, Phys Rev. E, 72(4), 045103, 2005

[5] E. Caglioti, N. Chernov and J. Lebowitz, Stability of solutions of hydrodynamic equations describing the scaling limit of a massive piston in an ideal gas, Nonlinearity, 17, 897-923, 2004.

[6] E. Caglioti and F. Rousset, Long time estimates in the mean field limit, preprint preprint is available at http://www.hyke.org/preprint/2004/15/157.pdf.

[7] E. Caglioti and F. Rousset, Quasistationary states for particle systems in the mean field limit, preprint 2005.

[8] E. Caglioti, P. L. Lions, C. Marchioro and M. Pulvirenti, A special class of stationary flows for two-dimensional Euler equation: a statistical mechanics description, Comm. Math. Phys. 143, 501-525, 1992.

[9] E. Caglioti, P. L. Lions, C. Marchioro and M. Pulvirenti, A special class of stationary flows for two - dimensional Euler equation: a statistical mechanics description: part II, Comm. Math. Phys., 174, 229-260, 1995.

[10] P. H. Chavanis, Kinetic theory of point vortices: diffusion coefficient and systematic drift, Phys. Rev. E 64, 026309, 2001.

[11] N. Chernov, J. L. Lebowitz and Ya. Sinai, Dynamic of a massive piston in an ideal gas, Russian Mathematical Surveys, 57, 1045-1125, 2002.

[12] N. Chernov, J. L. Lebowitz and Ya. Sinai, Scaling dynamic of a massive piston in a cube filled with ideal gas: exact results, Jour. Stat. Phys., 109, 529-548, 2002.

[13] N. Chernov, J. L. Lebowitz, Dynamics of a massive piston in an ideal gas: oscillatory motion and approach to equilibrium, Jour. Stat. Phys., 109, 507-527, 2002.

[14] E. Cagloti and C. Maffei, Time asymptotics for solutions of Vlasov-Poisson equation in a circle, Jour. Stat. Phys. 92(1-2), 301-3323, 1998.

[15] Ch. Gruber, Thermodynamics of systems with internal adiabatic constraints: time evolution of the adiabatic piston, Eur. J. Phys., 20, 259-266, 1999.

[16] Ch. Gruber and L. Frachebourg, On the adiabatic properties of a stochastic adiabatic wall: evolution, stationary non-equilibrium, and equilibrium states, Phys. A, 272, 392-428, 1999.

[17] J. Goodman. T. Hou and J. Lowengrub, Convergence of the point vortex method for the $2 D$ Euler equations, Comm. Pure Appl. Math., 43, 425-430, 1990.

[18] Ch. Gruber and J. Piasecki, Stationary motion of the adiabatic piston, Phys. A, 268, 412-423, 1999.

[19] Ch. Gruber, S. Pache and A. Lesne, Two times scale relaxation towards thermal equilibrium of the enigmatic piston, J. Stat. Phys., 112, 1199-1228, 2003.

[20] R. Holley, The motion of a heavy particle in an infinite one dimensional gas of hard spheres, Z. Wahrschein. verw. Geb., 17, 181-219, 1971.

[21] D. D. Holm, J. E. Marsden, T. Ratiu and A. Weinstein, Nonlinear stability of fluid and plasma equilibria, Phys. Rep., 123(1-2), 116, 1985.

[22] G. Joyce and D. Montgomery, Negative temperature states for the two-dimensional guidingcenter plasma, J. Plasma Phys. 10, 107, 1973. 
[23] E. Kestemont, C. Van den Broeck and M. Mansour, The "adiabatic" piston: and yet it moves, Europhys. Lett., 49, 143-149, 2000.

[24] Michael K. H. Kiessling, Statistical mechanics of classical particles with logarithmic interactions, Comm. Pure Appl. Math., 46, 1, 27-56, 1993.

[25] J. L. Lebowitz, J. Piasecki and Ya. Sinai, Scaling dynamics of a massive piston in an ideal gas, Hard Ball Systems and the Lorentz Gas, Encycl. Math. Sci., Springer, Berlin, 101, 217-227, 2000.

[26] E. Lieb, Some problems in statistical mechanics that I would like to see solved, Phys. A, 263, 491-499, 1999.

[27] T. S. Lundgren and Y. B. Pointin, Statistical mechanics of two-dimensional vorticies, J. Stat. Phys., 17, 323, 1977.

[28] C. Marchioro and M. Pulvirenti, A note on the stability of a spatially symmetric Vlasov-Poisson flow, Math. Meth. Appl. Sci., 8, 284-288, 1986.

[29] C. Marchioro and M. Pulvirenti, Mathematical Theory of Incompressible Nonviscous Fluids, Appl. Math. Sci., Springer-Verlag, New York, 96, 1994.

[30] M. Malek Mansour, C. Van den Broeck and E. Kestemont, Hydrodynamic relaxation of the adiabatic piston, Europhys. Lett., 69(2), 510-516, 2005.

[31] A. I. Neishtadt and Y. G. Sinai, Adiabatic piston as a dynamical system, J. Stat. Phys., 116, 1, 815-820,

[32] L. Onsager, Statistical hydrodynamics, Nuovo Cimento Suppl., 6, 279, 1949.

[33] J. Piasecki and Ch. Gruber, From the adiabatic piston to macroscopic motion induced by fluctuations, Phys. A, 265, 463-472, 1999.

[34] G. Rein, Non-linear stability for the Vlasov-Poisson system - the energy-Casimir method, Math. Meth. Appl. Sci., 17(14), 1129-1140, 1994.

[35] Y. G. Sinai, Dynamics of a massive particle surrounded by a finite number of light particles, Theor. Math. Phys., 125, 1351-1357, 1999.

[36] H. Spohn, Large Scale Dynamics of Interacting Particles, Texts and Monographs in Physics, Springer Verlag, 1991.

[37] Y. Y. Yamaguchi, J. Barré, F. Bouchet, T. Dauxois and S. Ruffo, Stability criteria of the Vlasov equation and quasi-stationary states of the HMF model, Physica A, 337, 36-66 2004. 